\title{
Predicting Outcomes of Active Sessions Using Multi-action Motifs
}

\author{
Weiqiang Lin \\ International Doctoral Innovation Centre \\ University of Nottingham \\ Ningbo, China \\ wyatt.lin@nottingham.edu.cn \\ Ke Zhou \\ School of Computer Science \\ University of Nottingham \\ Nottingham, United Kingdom \\ ke.zhou@nottingham.ac.uk
}

\author{
Natasa Milic-Frayling \\ School of Computer Science \\ University of Nottingham \\ Nottingham, United Kingdom \\ Natasa.Milic-Frayling@nottingham.ac.uk \\ Eugene Ch'ng \\ NVIDIA Joint-Lab on Mixed Reality \\ University of Nottingham \\ Ningbo, China \\ eugene.chng@nottingham.edu.cn
}

\begin{abstract}
Web sites and online services increasingly engage with users through live chats to provide support, advice, and offers. Such approaches require reliable methods to predict the user's intent and make an informed decision when and how to intervene during an active session. Prior work on predicting purchase intent involved clickstream data mining and feature construction in an ad-hoc manner with a moderate success (AUC 0.70 range). We demonstrate the use of the consumer Purchase Decision Model (PDM) and a principled way of constructing features predictive of the purchase intent. We show that the Logistic Regression (LR) classifiers, trained with multi-action motifs, perform on par with the state-of-the-art LSTM sequence model achieving comparable AUC ( 0.95 vs 0.96$)$ and performing better for the sparse purchase sessions, with higher recall ( 0.85 vs 0.61$)$ and higher F1 score ( 0.73 vs 0.66 ). While LSTM performs better than LR in terms of weighted averages of F1, precision, and recall, it requires 7 times longer to train and offers no insights about the predictive model in terms of the user actions and the purchase decision stages. The LR predictors are robust and effective in simulating real-time interventions, achieving F1 of 0.84 and AUC of 0.85 after observing only $50 \%$ of an active session. For non-purchase sessions that leaves room for live intervention, on average within 8 actions before the session ends.
\end{abstract}

\section{CCS CONCEPTS}

- Information system $\rightarrow$ Misc.

\section{KEYWORDS}

User behavior, Action motifs, User modelling, Consumer e-purchase, Purchase sessions

Permission to make digital or hard copies of part or all of this work for personal or classroom use is granted without fee provided that copies are not made or distributed for profit or commercial advantage and that copies bear this notice and the full citation on the first page. Copyrights for third-party components of this work must be honored

For all other uses, contact the owner/author(s).

WI'19, October 14-17, 2019, Thessaloniki, Greece

(c) 2019 Copyright held by the owner/author(s).

ACM ISBN 978-1-4503-6934-3/19/10.

https://doi.org/10.1145/3350546.3352495

\section{ACM Reference Format:}

Weiqiang Lin, Natasa Milic-Frayling, Ke Zhou, and Eugene Ch'ng. 2019. Predicting Outcomes of Active Sessions Using Multi-action Motifs. In IEEE/WIC/ACM International Conference on Web Intelligence (WI'19), October 14-17, 2019, Thessaloniki, Greece. ACM, New York, NY, USA, 9 pages. https://doi.org/10. $1145 / 3350546.3352495$

\section{INTRODUCTION}

Advances in Web technologies are enabling new modes of interactions between Web services and users. In particular, communication with clients through live chats has become a common practice in customer support, marketing and sales $[1,16]$. According to the American Market Association, customers who chat are 3 times more likely to make a purchase [29]. However, adoption of pro-active chat services is still relatively low, not exceeding $26 \%$ across industries [1]. Untimely attempts to connect with a customer present risks, potentially causing dissatisfaction due to unwarranted disruptions and increased privacy intrusion [3, 7]. The same applies to other forms of real-time interventions such as pop-up offers, advertisements and recommendations [35]. The effects are magnified in live interventions as they are more time consuming and more costly compared to presenting offers or help on the screen. Thus, it is critical to make a well-informed decision of when and how to intervene.

Existing literature on monitoring and intervening in users' activities covers a wide range of scenarios supported by mining clickstream data. The use scenarios range from optimizing search and navigation [10, 12, 21, 24, 39] to delivering contextual advertisements [40] and recommending related content and products [38, 42]. Clickstreams of registered users can include detailed history and personal preferences. For example, recent research predicts the likelihood of sales based on a consumer behavior over time [23] by creating an extensive user profile and observing user interactions with specific products in specific contexts [38]. However, many Web services do not or cannot collect historical personal data [3, 7]. Thus, a user needs to be assisted during an active session without prior knowledge about the user or the content that the user has accessed or created during the session.

Prior studies considered general session features such as click statistics, session duration and dwell time [28, 30, 42], or a few 


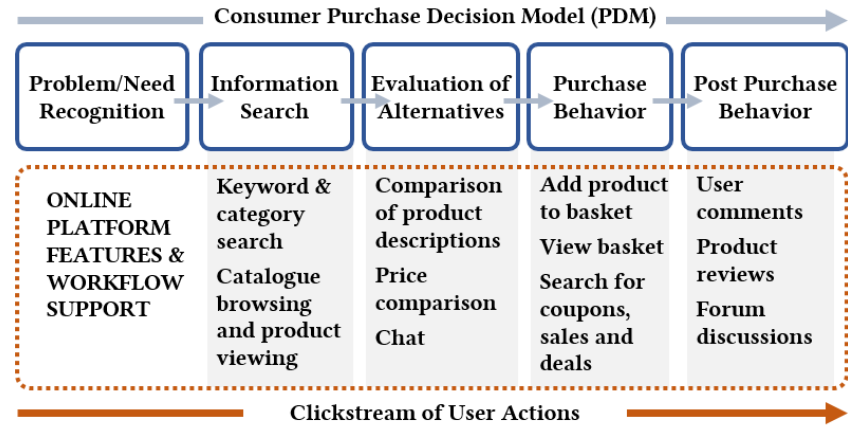

Figure 1: Five-stage sequential consumer Purchase Decision Model (PDM) [19] supported by e-commerce platform features

types of user actions such as product views [30]. We propose a systematic approach to e-commerce clickstream analysis aligned with the hypothesis:

An e-commerce site is designed to enable a purchase workflow, supporting user activities towards a product purchase. The clickstream captures the user's progress through the purchase decision stages and the action patterns within a session are indicative of the purchase outcome.

Designs of contemporary e-commerce platforms are optimized to assist the consumers with product purchases and are aligned with the consumer Purchase Decision Model (PDM) [19, 20] (Fig. 1). PDM considers a consumer's progression from identifying a need and searching for information to exploring alternatives, finalizing product selections and making a purchase. Thus action motifs, comprising sequences and sets of user actions related to PDM, are likely to capture the state of the user's purchase decision $[19,20]$ (Fig. 1). Predicting purchase outcomes of an active session presents a particular challenge since only an initial and, possibly, a small number of actions are observable. At the same time, insights about the predictive models and predictive features are highly desirable in order to make an informed decision when and how to intervene.

We demonstrate that the Logistic Regression (LR) classifiers based on action motifs are superior to those based on general session properties or individual user actions [30, 36]. They perform on par with the state-of-the-art Long Short Term Memory (LSTM) model for sequence processing (Section 5), achieving comparable AUC of 0.95 vs 0.96 . For the sparse purchase sessions, LR achieves higher recall of 0.85 vs 0.61 and F1 score of 0.73 vs 0.66 for LSTM.

The LR performance improves as we include more detailed user actions, e.g., break down search actions into search for categories vs general keyword search. Our experiments include RecSys [2], a publicly available e-commerce clickstream with limited action types, i.e., only product views. We demonstrate that the RecSys dataset is insufficient for exploring action-modeling in depth and experimenting with session predictions. We show that detailed clickstreams from JD.com e-commerce platform cover the entire purchase workflow and lead to effective predictors of session outcomes. Our detailed investigations led to key contributions:
(1) A principled and generalizable approach to clickstream mining using behavioural models to derive multi-action motifs. To the best of our knowledge, our study is the first to involve comprehensive clickstreams with user actions covering the full consumer purchase cycle.

(2) High LR prediction of purchase outcomes based on action motifs, achieving F1 of 0.91 and AUC of 0.95 and outperforming baseline predictors based on general session features and single action features. Using action motifs, LR performs on par with the LSTM sequence model with AUC of 0.95 vs 0.96 and weighted average F1 of 0.91 vs 0.95 .

(3) High LR performance for active sessions, achieving F1 of 0.84 and AUC of 0.85 for only $50 \%$ observed actions. This enables live interventions within remaining actions (on average, 8 actions for non-purchase sessions). The leading predictive features provide insights about possible interventions.

The latter is particularly significant as it provides foundations for live interventions to improve convergence in product sales. JD data shows that $40 \%$ of non-purchase sessions close within 5 minutes and the average number of actions for non-purchase sessions is 16.3. Furthermore, most of the product purchases $(70 \%)$ are completed within a single session, i.e., the purchased products have not been considered in prior sessions over at least one month. This implies that the consumers are not likely to re-engage with the same product purchase multiple times and, therefore, a timely intervention is key to converting users into buyers.

In the following sections we first discuss the related work and then formulate the problem and describe the methodology, experiments and findings. We conclude with the summary of the results and plans for future work.

\section{RELATED WORK}

In this section we reflect on research relevant to our work, focusing on feature construction and intent prediction from clickstream data and on consumer behaviour models in e-commerce.

Clickstream mining and motifs. Clickstream mining has been used extensively to support optimization of Web services, from predicting user browsing patterns [21] to detecting search intent and anticipating user queries $[10,12]$. Most scenarios related to the search intent predictions require extensive use of content features and user modeling based on general session statistics, clicks and use of navigation features [10]. By considering users' actions, Guo et al. [12] used interaction data to predict the goals of Web searchers.

Furthermore, research has been concerned with encoding of clickstreams and network graphs that arise in online communication and social media. Structural motifs have been used to study properties of network graphs that emerge from social interactions and content sharing in social media [4,27]. The shapes of network motifs are determined by communication events, e.g., replying to or forwarding messages. The basic motifs involve hops, splits and loops and can be used to represent complex network propagation [4]. In the case of social media, the motifs are used to characterize user types and community networks [41] and explain the graph propagation phenomena $[4,27]$. In our research, we identify action motifs from e-commerce clickstream data that captures the 
purchase workflow. We demonstrate their effectiveness in characterizing the user behaviour and predicting the purchase intent.

Predicting user intent. Most of the existing work in e-commerce aims at estimating conversion rates in relation to individual products, specific consumers, or combinations of the two [22, 25, 28, 32, 36, 42]. In particular, the Web search data [23] and clickstream data from e-commerce platforms, such as RecSys 2015, have been used to predict purchases $[25,28,32,36]$ and identify features that are good predictors, such as page visits [26, 36]. Yeo at al. [42] expanded the set of session features to include browsing activities and time spent on reviewing online product information. Furthermore, techniques like sequence mining and association rules have been applied to detect customers' purchase intent and determine probabilities of online purchases. online purchase intention $[18,33]$.

Our work extends this research by encoding clickstream data with a comprehensive set of action types, thus increasing the complexity of motifs detection, and by predicting purchase outcomes during an active sessions, based on limited information and a small number of users actions.

Modeling consumer behavior. Prior computational studies in ecommerce have adopted ad-hoc and brute-force feature engineering, aiming to uncover factors that explain consumer behavior, particularly the intent to buy [23, 25, 28, 32]. For example, Park et al. [28] adopted an extensive feature generation approach by incorporating session properties and combining session features. Other studies leverage detailed information about consumer characteristics [25, 43]. Among commonly used information are user demographics, including age, gender, and socio-economic status [23], and user purchase patterns and interests [17]. Similarly, information about past product purchases, product characteristics [25] and product reviews [43] are used to predict specific product sales. Studies commonly collect data from online shopping platforms or external services, such as search and social media, but lack a principled way for approaching behaviour modeling and optimizing e-commerce platform operations and customer support.

Our approach is well aligned with the conceptual framework of Butler \& Peppard [8] and the classic works on PDM by Ross [31] and Howard \& Jagdish [15]. By clearly specifying the user action set we systematically explore composite features, i.e., action motifs, that use them as building blocks. The PDM sequential stages naturally fit the multi-action sequences and action sets and associations among them [5, 6, 9, 37]. The high predictive performance of our action motifs empirically validates the utility of the PDM and confirms that PDM principles explain the online shopping behavior.

\section{BACKGROUND \& PROBLEM FORMULATION}

\subsection{Platform clickstreams}

E-commerce platforms like JD.com maintain detailed records of users' interactions with the system. For this research, JD provided user session data for the period of 1-31 August, 2016 in the form of Activity logs and Purchase logs. For each user, Activity logs capture the user's interactions with the platform, starting with logging in and out, which determine the start and the end of a shopping session. The corresponding Purchase logs record the date and time of each purchase made by the user. In order to determine whether a purchase occurred within a session, we aligned the Purchase and Activity logs. A session with at least one product purchase is designated as a purchase session; others are labelled as non-purchase sessions.

We also use RecSys Data [2], a publicly available clickstream of shopping activities over a period of six months. Each entry in the clickstream is designated by a Session ID, Time Stamp, Product ID and Product Category ID and relates to viewing a product page. Entries with the same Session ID are collected to create a session. The start of a session is approximated by the time-stamp of the first product viewing within a given session ID. The end of the session is approximated by considering the average time difference between viewing actions and adding it to the time of the last action in the session.

Table 1: Definition of action types in 8-APM and 14-APM; 3-APM features are designated by asterix $\left(^{*}\right)$

\begin{tabular}{|c|c|c|c|}
\hline PDM phase & Definition & 8-APM & 14-APM \\
\hline \multirow{5}{*}{$\begin{array}{l}\text { Information } \\
\text { search }\end{array}$} & Keyword search & \multirow{2}{*}{ S } & KS \\
\hline & Category search & & CS \\
\hline & Item view & \multirow{3}{*}{$\mathrm{V}$} & $\mathrm{IV}^{*}$ \\
\hline & Repeated view & & $\mathrm{RV}^{*}$ \\
\hline & Order history view & & OV \\
\hline Evaluation of & Comparison & $\mathrm{C}$ & $\mathrm{C}^{*}$ \\
\hline Alternatives & Chat & CHAT & CHAT \\
\hline \multirow{5}{*}{$\begin{array}{l}\text { Purchase } \\
\text { Behavior }\end{array}$} & Add to basket & \multirow{2}{*}{ B } & $\mathrm{AB}$ \\
\hline & View basket & & VB \\
\hline & Coupons & \multirow{3}{*}{$\mathrm{D}$} & $\mathrm{SC}$ \\
\hline & Sales info. & & SI \\
\hline & Specific deals & & SS \\
\hline $\begin{array}{c}\text { Post Purchase } \\
\text { Behavior }\end{array}$ & Comments & CMT & CMT \\
\hline Other & $\begin{array}{c}\text { Home page visits } \\
\text { Logging in \& out } \\
\text { Password change, etc. }\end{array}$ & $\mathrm{O}$ & $\mathrm{O}$ \\
\hline
\end{tabular}

Focusing on user actions, we process JD Activity logs to create two annotated clickstreams: one using 8 action types, referred to as 8-Action Purchase Model (8-APM), and the other using 14 action types (14-APM). Table 1 lists action types and groups them into four PDM stages [8, 15, 31] (Fig. 1). User actions that are not strictly related to PDM stages, such as visiting JD home page, logging in and out, managing the personal shopping account and password, checking finances and online trading accounts, etc., are grouped under the category O. Since RecSys data includes only product viewing (V), we construct a simple 3-Action Purchase Model (3APM) that can be used across both data sets; 8-APM and 14-APM apply to JD data only.

In some instances the session may include multiple purchase orders. Even though the sessions do not include actions of ordering and payment, we still want to make sure that actions following the purchase order do not reveal that the purchase occurred and therefore affect the testing of our algorithms. Thus, we eliminate all the actions that follow the first purchase. Thus, all the purchase sessions (top session in Fig. 2) end with the action performed right before initiating the purchase. Non-purchase sessions (bottom session in Fig. 2) contain all user actions. 


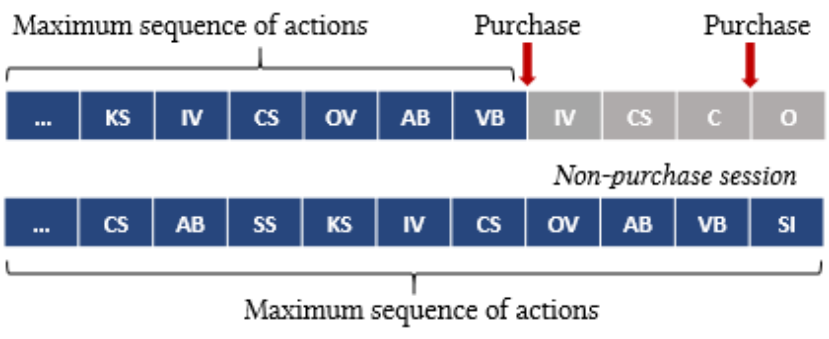

Figure 2: Representation of purchase and non-purchase sessions with actions removed after the first purchase order

\subsection{Session Representations \& Action Motifs}

We model user sessions using features derived from the action sets and session statistics:

- General session features (GenF) are motivated by prior work $[9,31]$ that considers purely session related parameters. The session parameters typically include session duration, number of user clicks, average time per click, and similar. In the case of JD data, we also include average time and total time spent on each action type within a given session.

- Three-Action Purchase Model (3-APM) includes three actions related to acquiring information about a product and evaluating alternatives: Item View (IV), Repeated View (RV) and Comparison (C).

- Eight-Action Purchase Model (8-APM) includes 8 actions related to acquiring information, evaluating alternatives, and deciding whether to make a purchase: Search (S), View (V), Basket interaction (B), Comparison (C), Deal (D), Chat (CHAT), Comments (CMT) and Other (O) (Table 1).

- Fourteen-Action Purchase Model (14-APM) is a refinement of 8-APM (Table 1) with more detailed actions. Deals (D) are broken down into looking for general coupons (SC) and sales discounts (SI) and specific product deals (SS). Basket interaction (B) is refined to differentiate between add-tobasket $(\mathrm{AB})$ and visit basket $(\mathrm{VB})$ actions.

From the clickstreams annotated with 8-APM and 14-APM action types (Section 3.2 and Table 1), we identify multi-action motifs, i.e., action sequences ActSeq and action sets ActSet. More precisely, for a sequence of action types $a_{t}$ in a session, $a_{t} \in\left\{a_{1}, a_{2}, \ldots, a_{p}\right\}$ we define ActSeq motifs as permutations with repetitions of action types $a_{t}$. Similarly, we define ActSet motifs as any combination (without repetitions) of action types $a_{t}$. Besides the frequencies of individual motifs we also consider associations among motifs. This led to three sets of features:

- Action Sequence Motifs. For an annotated clicksteam, comprising user sessions $\mathrm{s} \in \mathrm{S}, \mathrm{s}=\left(a_{1}, a_{2}, \ldots, a_{n}\right), a_{i} \in A$, we adapt the method by Wang et al. [37] to generate ActSeq motifs by mining frequent subsequences across sessions in $\mathrm{S}$. The resulting collection of action sequence motifs is referred to as FreqActSeq. For each session, we record the presence or absence of a given ActSeq motif.

- Action Set Motifs. For an annotated clickstream, comprising user sessions $\mathrm{s} \in \mathrm{S}, \mathrm{s}=\left(a_{1}, a_{2}, \ldots, a_{n}\right), a_{i} \in A$, we adapt the frequent item-set mining algorithm by Borgelt $[5,6]$ to identify subsets of actions $A_{m} \subseteq A$ that are found at least $f$ times among the observed sessions. We set $f$ to $50 \%$ of purchase sessions. The resulting collection of action set motifs is referred to as FreqActSet. For each session we record the presence or absence of a given ActSet motif.

- Association Rules. We adopt the method by Chen et al. [9] to detect associations among sets of session actions. For two action sets $A_{1}, A_{2} \subset A, A_{1} \cap A_{2}=\emptyset$, we derive an association $A_{1} \Rightarrow A_{2}$ with the minimum threshold thre $\left(A_{1} \Rightarrow A_{2}\right)$ and confidence conf $\left(A_{1} \Rightarrow A_{2}\right)$ which reflect the number of sequences that contain $A_{1}$ and, among them, the percentage of sequences that also contain $A_{2}$, respectively. We set the minimum threshold to 0.50 of total sessions and the confidence rate at 0.85 . We refer to the resulting set of features as Action Association Rules, designated by ActAssocR. For each session, we record the presence or absence of a given association rule.

\subsection{Classification \& Real-time Predictions}

Session features and action motifs are used to train Logistic Regression (LR) [14] classifiers to discern between purchase and nonpurchase sessions. All our experiments use 10-fold validation, measuring F1 score (for individual classes and the weighted average for two classes) and AUC. LR models with more detailed APMs consistently outperform classifiers using simpler action sets, including LSTM trained on the 14-AMP clickstream [25, 26, 36]. LSTM $[11,13]$ is capable of learning long-term (distant) dependencies in sequential data. That makes it an ideal baseline, allowing us to compare its performance with LR classifiers with multi-action motifs that aim to capture the action dependencies and progression along the PDM stages.

Furthermore, we formulate the real-time session prediction as a binary classification problem with evolving session data: for a given session $s$ and a given step $K$, in a session sequence we assign a label $l \in\{1,-1\}$ to the truncated session $s_{K}$ based on the observed actions $\left(a_{1}, a_{2}, \ldots, a_{K}\right)$, where $l=1$ designates $a$ purchase session and $l=-1$ a non-purchase session. (When beneficial, we also use $P$ for $l=1$ and $N P$ for $l=-1$.) The label assigned at step $K$ is interpreted as a prediction of whether or not a purchase will occur in the remainder of the session $\left(a_{K+1}, a_{2}, \ldots, a_{n}\right)$.

We run simulations of evolving sessions by evaluating the classifiers for $K$ actions where $K$ corresponds to a fixed percentage $N \%, N=10,20, \ldots, 100$ of the session duration. It is expected that the prediction accuracy will increase as a larger proportion of user actions is revealed to the predictor [23]. The key is to achieve a favorable trade-off between the accuracy and the time left for a possible intervention before the end-of-session $n$.

In practice, the session duration is not known. Predicting the number of actions in the session is an interesting problem but out of scope of this paper; here we focus on the effectiveness of action motifs in making accurate predictions of purchase intent with scarce data (as low as $10 \%$ of the session actions). Furthermore, one can envision the classification model being updated in real time as the session progresses. However, in a realistic scenario, the model is trained off-line and applied to a relatively large number of 


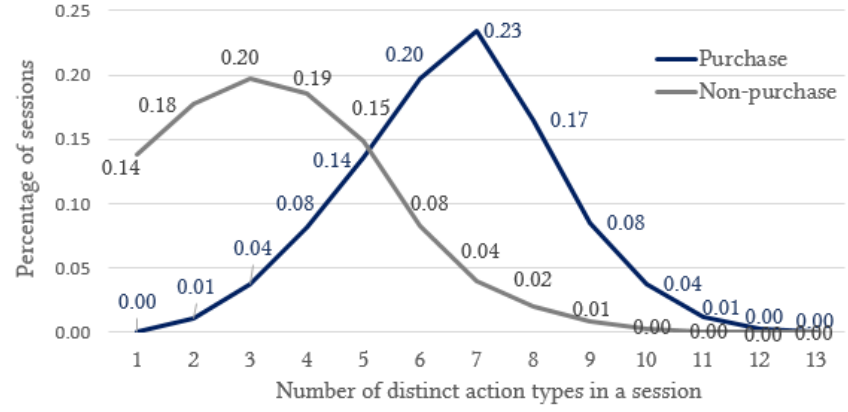

Figure 3: Distribution of distinct action types (14-APM) across purchase and non-purchase sessions in JDdata, showing a larger diversity of actions in purchase sessions

concurrent sessions. Thus, adjusting the model based on a single session evidence is interesting but not aligned with the use scenario.

\section{DATA \& SESSION ANALYSIS}

In our experiments we use two data sets: RecSys and JDdata. Basic statistics of the two data sets are provided in Table 2, showing that purchase sessions comprise only $7.5 \%$ of all the sessions in RecSys and $5.5 \%$ in JDdata.

Table 2: Data set statistics

\begin{tabular}{l|c|c}
\hline Statistics & RecSys & JDdata \\
\hline \hline Number of Users & - & 178,441 \\
\hline Number of Purchase Sessions & 509,696 & 297,496 \\
\hline Number of Non-purchase Sessions & $8,740,033$ & $3,674,657$ \\
\hline Total number of Sessions & $9,249,729$ & $3,972,153$ \\
\hline Total number of Actions & $33,003,944$ & $41,256,912$ \\
\hline
\end{tabular}

By analyzing a typical user workflow on the JD platform, we note that no purchases can be completed in a less than 5 steps: Login $\rightarrow$ Search/View $\rightarrow$ Select $\rightarrow$ Purchase $\rightarrow$ Pay. Therefore, in order to avoid any trivial classifiers of non-purchase sessions we filtered out sessions with fewer than 5 actions in JDdata. The remaining data set includes 297,496 purchase sessions and 1,485,408 non-purchase sessions.

Distribution of action types. In Fig. 3 we show the distribution of distinct action types from 14-APM across purchase and nonpurchase sessions, indicating higher diversity of action types in purchase sessions. The peak of the skewed distribution for nonpurchase session is at 3 distinct action types, present in $20 \%$ of sessions. In contrast, the peak for purchase sessions is at 7 distinct action types, present in $23 \%$ for purchase sessions. Thus, in purchase sessions the consumer has progressed further through the PDM stages $[19,20]$ and engaged in a broader range of actions, possibly including searching for deals, adding items to the basket and using chat to consult with the customer support.

Frequency of action types. Similarly, Fig. 4 shows the average frequency and percentages of 14-APM action types across purchase and non-purchase sessions in JDdata. As expected, purchase

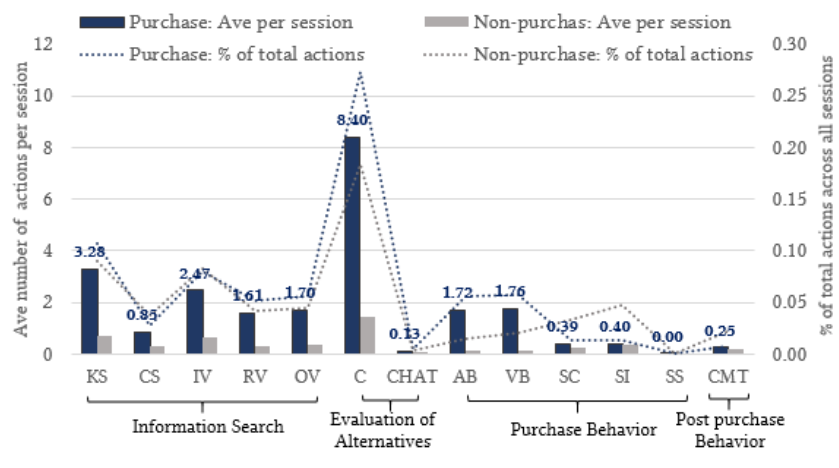

Figure 4: Average frequency and percentage of 14-APM action types per session, showing a higher prominence of actions in purchase sessions

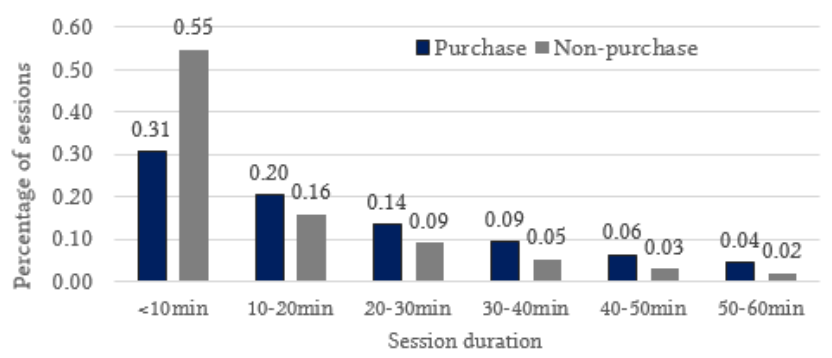

Figure 5: Distribution of session duration for purchase and non-purchase sessions for JDdata

sessions are more likely to include basket related actions (AddBasket (AB), View-Basket (VB)) and involve product comparisons (C). Indeed, nearly $60 \%$ of non-purchase sessions have no product comparison activities, in contrast to only $20 \%$ of purchase sessions. Similarly, $65 \%$ of non-purchase sessions contain no keyword search actions (KS) compared to only $22 \%$ of purchase sessions without keyword search. In fact, JDdata shows that the percentage of keyword search actions in purchase sessions is always higher than in non-purchase sessions, suggesting that more prominent information searching is aligned with the consumer's intent to buy.

Session duration. From Fig. 5 we see that $40 \%$ of non-purchase sessions close within 5 minutes, revealing a high rate of abandonment in the early stages. At the same time, $15 \%$ of purchase sessions complete within 5 minutes, indicating the clarity of the user's need and intent to purchase. Furthermore, 95\% of all shopping sessions take no longer than 3 hours and $93 \%$ of shopping sessions without purchases end within 3 hours. Most of the purchase sessions (73.1\%) involved a single product purchase and $95.9 \%$ sessions involve up to three product purchases.

For live interventions, it is important to know how many actions users perform during sessions. Considering sessions with more than 5 actions, we find that non-purchase sessions involve 16.3 user actions on average, with a standard deviation of 22.58 and a range between 5 and 5813. Purchase sessions involve 34 actions per 
session on average, with a standard deviation of 33.97 and a range from 5 to 2163 .

Action motifs. Our analysis of action motifs shows that multiaction sets and sequences are present in a large percentage of sessions and follow different distributions in purchase (P) and nonpurchase (NP) sessions. For example, for 8-APM, the sequence $(\mathrm{O}, \mathrm{V}, \mathrm{V})$ is found in $81 \% \mathrm{P}$ and $41 \% \mathrm{NP}$ sessions, $(\mathrm{V}, \mathrm{C})$ in $80 \% \mathrm{P}$ and $42 \% \mathrm{NP}$, and $(\mathrm{V}, \mathrm{V})$ in $87 \% \mathrm{P}$ and $50 \% \mathrm{NP}$ sessions. Generally, among the top 10 motifs for 8-AMP, 7 involve Viewing $(\mathrm{V})$ in combination with other features (top 10 8-AMP sequences: $(\mathrm{O}, \mathrm{V}),(\mathrm{O}, \mathrm{O})$, $(\mathrm{V}, \mathrm{V}),(\mathrm{V}, \mathrm{O}),(\mathrm{O}, \mathrm{O}, \mathrm{O}),(\mathrm{V}, \mathrm{B}),(\mathrm{O}, \mathrm{V}, \mathrm{V}),(\mathrm{V}, \mathrm{C}),(\mathrm{S}, \mathrm{V}),(\mathrm{O}, \mathrm{B}))$. A significant presence of $\mathrm{O}$ features is expected since they include logging in and out and accessing the JD home page. We expect the set and sequence motifs to capture the PDM stages in individual sessions and to differentiate well between $\mathrm{P}$ and NP sessions.

\section{CLASSIFICATION EXPERIMENTS}

In this section we present experiments that explore effects of feature construction on the performance of binary classifiers (into $\mathrm{P}$ and NP sessions) by considering (1) refined action types, from 3-APM and 8-APM to 14-APM and (2) combinations of action motifs. They all involve training and testing classifiers through 10 -fold validation and reporting F1-metric (weighted average) and AUC scores. We perform statistical significance tests (student t-test) for all the performance comparisons that are of interests.

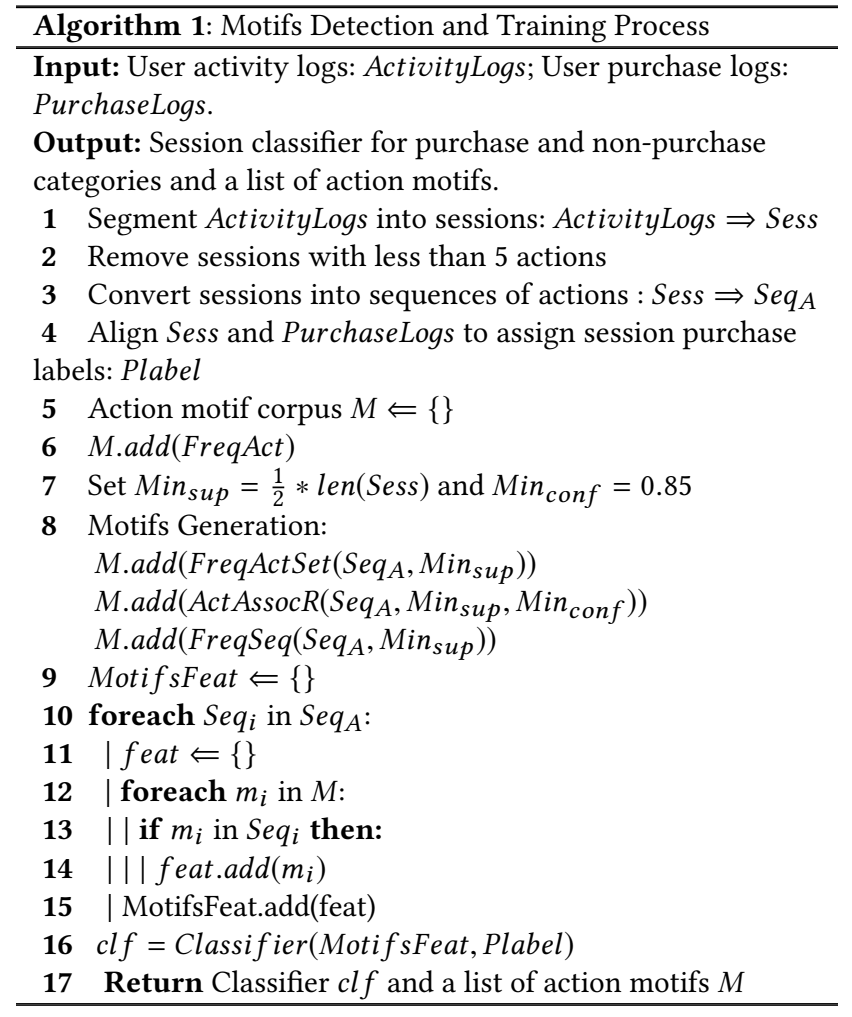

For comparing relative effectiveness of action features we consider two baseline LR classifiers trained with (a) general session features (GenF) and (b) individual action features (FreqAct), respectively. GenF differs for the RecSys and JDdata since the two clickstreams do not provide the same session information. They comparably cover session duration, total number of clicks in a session, total time of individual actions, average time of individual actions, and total number of clicks for item pages. Both types of features, GenF and FreqAct, have been explored in prior research. Van den Peol \& Buckinx [36] refer to them as 'general' and 'detail' features, respectively, and report AUC 0.696 on their data set [36]. Furthermore, we compare the best LR classifier with the LSTM model trained using 14-APM annotated clickstream to illustrate the benefits of the multi-action features constructed based on PDM.

\subsection{Comparison of APMs and Feature Sets}

We first consider 3-APM LR classifiers that allow us to compare performance on both RecSys and JDdata. Since 8-APM and 14-APM cannot be applied to RecSys data, 3-APM experiments enable us to assess a relative 'difficulty' of session classification on the two data sets. LR classifiers deal with limited action information in the RecSys data and a significant class imbalance in JDdata. Using AUC scores makes the evaluation more robust with regards to the class imbalance.

5.1.1 Experiments with 3-APM across Data Sets. Performance statistics in Table 3 shows that 3-APM LR classifiers achieve similar performance for both Recsys and JDdata. With only three actions to consider, the multi-action features do not have a benefit of capturing the progression in the purchase process. Indeed, considering individual features FreqAct achieves AUC of 0.71 and 0.70 on RecSys and JDdata respectively while a combination with multi-action sequences $(\mathrm{f} 1+\mathrm{f} 2+\mathrm{f} 3)$ achieves AUC of 0.70 on both data set. The general session features GenF perform comparably well with AUC of 0.72 for RecSys and AUC of 0.71 for JDdata.

5.1.2 Experiments with 8-APM and $14 A P M$. Considering highly imbalanced $\mathrm{P}$ and NP classes in JDdata, we train LR classifiers on a balanced subset of JDdata and test on the imbalanced data set. LR classifiers with general session features (GenF) achieve F1 0.78 and AUC 0.80 score (Table 3) and set a baseline for action-based features. Action motifs e.g., FreqActSeq, consistently outperform individual action features (FreqAct) for both 8-APM (F1: 0.90 vs 0.85 , AUC: 0.93 vs 0.87 ) and 14-APM (F1: 0.91 vs 0.85 , AUC: 0.94 vs 0.90) (Table 3).

LR performance with FreqActSeq motifs for 8-APM and 14-APM shows improvement of $5 \%$ and $6 \%$ in $\mathrm{F} 1$ measure and $6 \%$ and $4 \%$ in AUC scores, respectively, compared to an already high baseline that FreqAct features offer (F1 0.85 and AUC 0.90). These performance differences are statistically significant.

According to Fig. 6, LR performs best for combinations of FreqActSeq motifs with other feature sets, particularly with FreqActSet motifs. These results confirm our hypotheses that multi-action motifs may capture action dependencies and the user's progression through the purchase decision stages. Furthermore, we observe that the highest LR coefficients associated with features of FreqActSet and FreqActSeq classifiers are associated with multi-action set and sequence motifs (Section 5.2).

The experiments also clearly demonstrate that more refined action model 14-APM consistently produces better classifiers than 
Table 3: Performance of LR classifiers with features generated from 3-APM, 8-APM and 14-APM action sets

\begin{tabular}{l|c|c|c|c|c|c|c|c}
\hline \multirow{2}{*}{ Feature Set } & \multicolumn{2}{|c|}{ RecSys Data } & \multicolumn{6}{|c}{ JDdata } \\
\cline { 2 - 10 } & \multicolumn{2}{|c|}{ 3-APM } & \multicolumn{2}{|c}{ 3-APM } & \multicolumn{2}{c}{ 8-APM } & \multicolumn{2}{|c}{ 14-APM } \\
\cline { 2 - 10 } & F1 & AUC & F1 & AUC & F1 & AUC & F1 & AUC \\
\hline \hline GenF (GF) & 0.80 & 0.72 & 0.69 & 0.71 & 0.78 & 0.80 & 0.78 & 0.80 \\
\hline FreqAct (f1) & 0.85 & 0.71 & 0.74 & 0.70 & 0.85 & 0.87 & 0.85 & 0.90 \\
\hline FreqActSet (f2) & 0.76 & 0.67 & 0.58 & 0.64 & 0.87 & 0.88 & 0.90 & 0.92 \\
\hline ActAssocR (f3) & 0.76 & 0.67 & 0.58 & 0.64 & 0.87 & 0.88 & 0.90 & 0.92 \\
\hline FreqActSeq (f4) & 0.76 & 0.67 & 0.69 & 0.69 & 0.90 & 0.93 & 0.91 & 0.94 \\
\hline f1+f2+f4 & 0.75 & 0.70 & 0.69 & 0.70 & 0.90 & 0.93 & 0.91 & 0.95 \\
\hline GF+f1+f2+f4 & - & - & - & - & 0.90 & 0.93 & 0.91 & 0.94 \\
\hline GF+f1+f2+f3+f4 & - & - & - & - & 0.90 & 0.93 & 0.91 & 0.95 \\
\hline
\end{tabular}

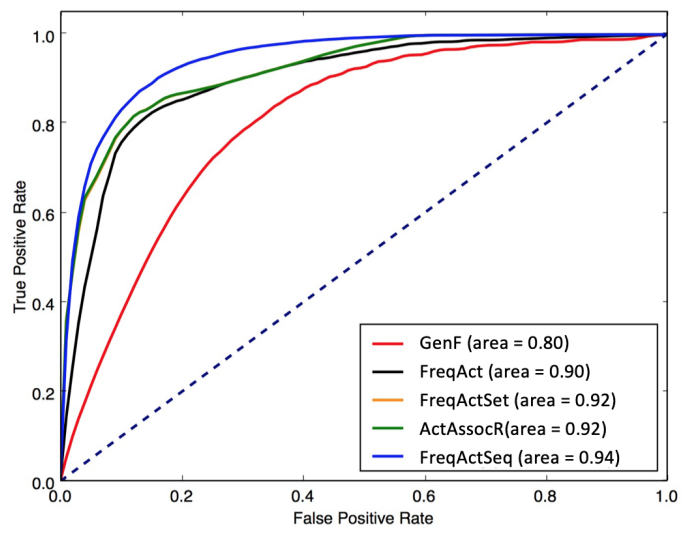

Figure 6: AUC curves for LR classifiers trained with 14-AMP features

8-APM across individual motif types ( $\mathrm{f} 1, \mathrm{f} 2, \mathrm{f} 3, \mathrm{f} 4)$ and their combinations. For example, 14-APM FreqActSeq combined with other feature types outperform the same 8-APM combinations $(\mathrm{f} 1+\mathrm{f} 2+\mathrm{f} 4$ AUC 0.95 vs 0.93 ) and the difference in performance is statistically significant (Table 3).

We note that the performance difference between action motifs FreqActSet, and association rules ActAssocR is not statistically significant and we omit the latter from our further analysis.

\subsection{Comparison with LSTM Model}

We use the LSTM model to assess the relative effectiveness of the feature construction based on PDM in capturing action dependencies and purchase decision stages. We compared 14-APM LR classifiers to the LSTM model trained on the 14-APM JDdata clickstream with no feature engineering. In our experiments, the LSTM hidden layer size is set to 128 . The weight matrices are initialized randomly and the bias vectors are initialized to zero. We use RMSprop [34] optimizer, a sigmoid nonlinear activation function, and data batches of size 1024 for up to 25 epochs (Table 2). Inputs are vectors representing sequences of individual actions and the outputs are individual session predictions as $\mathrm{P}$ or NP.

Table 4 shows that the AUC performance of LR $(\mathrm{f} 1+\mathrm{f} 2+\mathrm{f} 4)$ and LSTM classifiers is comparable in terms of AUC (0.95 vs 0.96$)$ as well as the precision for NP session (0.97 vs 0.97 ). However, LR
Table 4: Performance of the LR and LSTM models for 14-APM features, with weighted averages (AVG) of performance metrics for $P$ and NP classes

\begin{tabular}{l|c|c|c|c|c|c|c|c|c|c}
\hline \multirow{2}{*}{ Features } & \multirow{2}{*}{ AUC } & \multicolumn{3}{|c|}{ Precision } & \multicolumn{3}{c|}{ Recall } & \multicolumn{3}{c}{ F1 } \\
\cline { 3 - 11 } & & NP & P & AVG & NP & P & AVG & NP & P & AVG \\
\hline \hline LR(f1+f2+f4) & 0.95 & 0.97 & 0.64 & 0.91 & 0.90 & 0.85 & 0.90 & 0.94 & 0.73 & 0.91 \\
\hline LSTM & 0.96 & 0.97 & 0.72 & 0.95 & 0.98 & 0.61 & 0.96 & 0.98 & 0.66 & 0.95 \\
\hline
\end{tabular}

performs better for the sparse $\mathrm{P}$ sessions, with higher recall $(0.85$ vs 0.61 ) and higher F1 score ( 0.73 vs 0.66 ). The precision for the NP class is particular important since interventions are likely to focus on turning NP activities into purchases and selecting NP sessions reliably, with high precision, is essential.

Although weighted averages AVG for LSTM are higher than for LR (precision 0.95 vs 0.91 , recall 0.96 vs 0.90 and F1 0.95 vs 0.91 ), the LSTM method is computationally more expensive (7 times longer to train than LR with complex features). This is particularly important for live interventions, in case the model needs to be updated on a continual basis. Finally, LSTM does not offer any insights about features of the predictive model in terms of the user actions and purchase stages. A closer inspection of the LR classifiers show that the leading predictive features (Top 5) are FreqActSet $\{\mathrm{IV}, \mathrm{O}\}$ and FreqActSeq (IV,O,O), (IV,O), (IV,VB),(O,O,O). The presence of ItemView and BasketView indicates that the user has progressed through the stages and therefore, it might be appropriate to intervene with suggestions for additional products or by placing ads on the basket pages.

\section{REAL-TIME PURCHASE PREDICTIONS}

Real-time session interventions require highly effective session classifiers to predict session outcomes (P or NP) from actions observed in an evolving session. It is desirable to have high recall for the sparse $\mathrm{P}$ sessions and high precision for the abundant NP sessions. In view of the lower LSTM performance for $\mathrm{P}$ sessions in terms of Recall and F1 (Table 4), we present our experiment analysis for the LR classifiers. We explore trade-offs between precision achieved for partial sessions and the number of remaining action steps that provide an opportunity for intervention. Due to the space limitation, we discuss results only for the best LR classifier trained with 14-APM features: FreqAct+FreqActSet+FreqActSeq (Table 3). For a given trained classifier, we simulate classification of live sessions:

(1) For a collection $T$ of test sessions $s$ and given $N, N=10, \ldots, 100$, consider $\mathrm{N} \%$ of session actions in $s$, i.e., a truncated session $s_{N}=\left(a_{1}, a_{2}, \ldots, a_{j}\right)$, where $j=N \% * n, n$ is the session length in terms of the number of actions. Generate 14-APM motifs and represent the session using a combination of Fre$q A c t+$ FreqActSet+FreqActSeq features.

(2) For each step $\mathrm{k}, \mathrm{k}=1, \ldots, N \% * n$, apply the trained LR classifier and assign a label to the sub-session $\left(a_{1}, a_{2}, \ldots, a_{k}\right)$. The process continues until the classifier assigns the purchase label $P$ or reaches the end of the sub-session $\mathrm{j}=N \% * n$.

The pseudo code is shown in the description of Algorithm 2.

Simulation experiments are performed using a test set comprising 29,270 P and 148,540 NP sessions. Performance statistics in Table 5 shows consistent improvement of LR performance in terms 


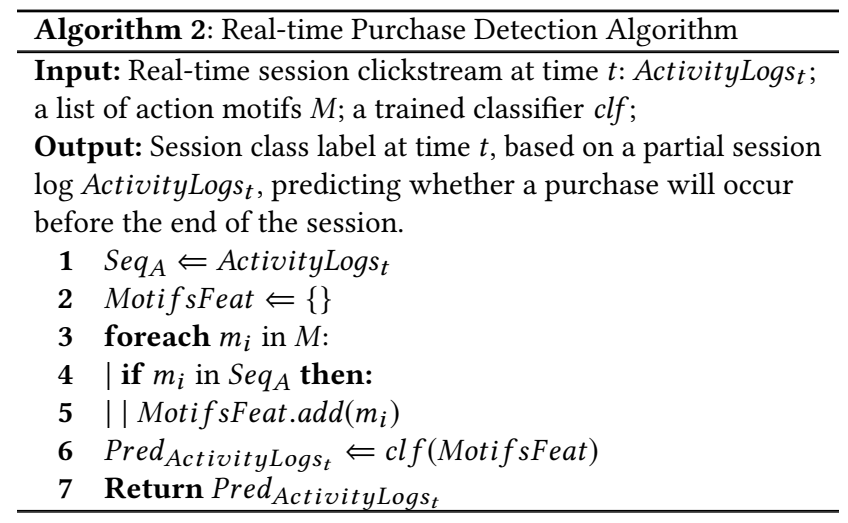

of F1, Accuracy and AUC, with the increased percentage N\% of session actions. Applying the cut off level of 50\% results in F1 0.84 and AUC 0.85 and leaves, on average, 8 remaining actions before a typical NP session ends and, on average, 17 actions in $\mathrm{P}$ sessions (Table 5). Even after $90 \%$ of session actions are observed there are, on average, 2-3 remaining actions available to begin a live interaction while leveraging high LR performance of F1 0.88 and AUC 0.93 .

Table 5: Average number of actions per truncated test sessions $s_{N}$ and the corresponding LR performance

\begin{tabular}{c|c|c|c|c|c|c}
\hline \multirow{2}{*}{ Percent } & \multicolumn{3}{|c|}{ Average Num of Actions } & \multicolumn{3}{c}{ LR Performance } \\
\cline { 2 - 7 } & P\&NP & P & NP & Accuracy & F1 & AUC \\
\hline \hline $10 \%$ & 1.4 & 3.0 & 1.1 & 0.84 & 0.77 & 0.70 \\
\hline $20 \%$ & 3.5 & 6.4 & 2.9 & 0.84 & 0.78 & 0.77 \\
\hline $30 \%$ & 5.2 & 9.8 & 4.4 & 0.85 & 0.80 & 0.79 \\
\hline $40 \%$ & 7.2 & 13.2 & 6.2 & 0.85 & 0.82 & 0.82 \\
\hline $50 \%$ & 9.2 & 16.8 & 7.9 & 0.86 & 0.84 & 0.85 \\
\hline $60 \%$ & 11.0 & 20.0 & 9.4 & 0.86 & 0.85 & 0.86 \\
\hline $70 \%$ & 12.9 & 23.4 & 11.0 & 0.86 & 0.86 & 0.88 \\
\hline $80 \%$ & 14.8 & 26.8 & 12.7 & 0.87 & 0.87 & 0.90 \\
\hline $90 \%$ & 16.7 & 30.2 & 14.3 & 0.88 & 0.88 & 0.93 \\
\hline $100 \%$ & 19.0 & 34.0 & 16.3 & 0.88 & 0.89 & 0.95 \\
\hline
\end{tabular}

We also consider LR classification of sessions truncated at a pre-set number of actions $r$, i.e., assuming that for a given session, intervention will occur after observing $r$ user actions. In Table 6 we show the performance for sessions longer than $r=10,16$, and 34, considering that 16 and 34 are average numbers of actions in NP and $\mathrm{P}$ sessions, respectively (Table 5). Increasing the cut-off level reduces the data set (to sessions longer than $r$ ) and, therefore, the results are not easily comparable. It is encouraging that the recall is improved for longer P sessions (recall of 0.71 for $r=34$ ), enabling better detection of $\mathrm{P}$ sessions in a highly imbalanced data set.

Live intervention opportunities. In contrast to LSTM, action-based models enable us to investigate purchase intent in more depth and identify interventions that may be appropriate and effective. We illustrate the approach by considering two types of actions: Deals that include search for coupons (SC), information (SI) and special deals (SS), and Basket actions such as viewing items in the basket (VB) and adding new items to the basket (AB) (Table 1).
Table 6: LR classifier performance for a pre-set number of actions in an active session

\begin{tabular}{c|c|c|c|c|c|c}
\hline Actions & Acc & $\operatorname{Prec}(\mathrm{NP})$ & $\operatorname{Prec}(\mathrm{P})$ & $\operatorname{Rec}(\mathrm{NP})$ & $\operatorname{Rec}(\mathrm{P})$ & $\mathrm{F} 1$ \\
\hline \hline 10 & 0.77 & 0.79 & 0.58 & 0.93 & 0.30 & 0.74 \\
\hline 16 & 0.74 & 0.78 & 0.62 & 0.85 & 0.50 & 0.73 \\
\hline 34 & 0.70 & 0.78 & 0.60 & 0.70 & 0.71 & 0.70 \\
\hline
\end{tabular}

Table 7 shows the proportion of Deal and Basket related actions in truncated $s_{N}$ sessions that retain $\mathrm{N} \%, \mathrm{~N}=10, \ldots, 90$ of the full session. With increasing N\%, the distribution of Deal actions increases faster for NP sessions than $\mathrm{P}$ sessions, reaching the proportion of 0.31 for NP $90 \%$ sub-sessions. For purchase sessions, searching for Deals is less prominent, reaching only 0.25 of actions in P $90 \%$ subsessions. Basket related activities are expected to be less prominent in NP session. Indeed, they represent 0.35 of activities in NP 90\% sub-sessions in contrast to 0.83 of activities in $\mathrm{P} 90 \%$ sub-sessions.

We conclude that customers involved in NP sessions are still in the information seeking and gathering stage, investigating options and exhibiting a degree of price sensitivity. A steady increase of Deal activities with increased N\%, suggests that intervening with Deals can be beneficial at any point in the session. However, it seems more important to assist the consumer with gathering product information and exploring options since these are prerequisites for making a purchase decision and placing an order.

Table 7: Proportion of Deal and Basket actions in truncated $P$ and NP sessions

\begin{tabular}{c|c|c|c|c}
\hline \multirow{2}{*}{ \%Session } & \multicolumn{2}{|c|}{ Purchase Session } & \multicolumn{2}{c}{ Non-purchase Session } \\
\cline { 2 - 5 } & Basket & Deal & Basket & Deal \\
\hline \hline $10 \%$ & 0.08 & 0.05 & 0.03 & 0.06 \\
\hline $20 \%$ & 0.19 & 0.08 & 0.08 & 0.14 \\
\hline $30 \%$ & 0.29 & 0.11 & 0.13 & 0.17 \\
\hline $40 \%$ & 0.40 & 0.13 & 0.18 & 0.20 \\
\hline $50 \%$ & 0.52 & 0.16 & 0.22 & 0.23 \\
\hline $60 \%$ & 0.63 & 0.18 & 0.26 & 0.25 \\
\hline $70 \%$ & 0.72 & 0.20 & 0.30 & 0.27 \\
\hline $80 \%$ & 0.80 & 0.23 & 0.33 & 0.29 \\
\hline $90 \%$ & 0.83 & 0.25 & 0.35 & 0.31 \\
\hline $100 \%$ & 0.83 & 0.26 & 0.37 & 0.33 \\
\hline
\end{tabular}

\section{CONCLUDING REMARKS}

In this paper we present a systematic approach to clickstream mining and feature construction with the goal to train reliable classifiers for predicting active session outcomes and supporting live interventions during an evolving user session. We demonstrate the effective use of action motifs to capture the user purchase intent.

Logistic Regression (LR) classifiers trained with action motifs comprising sets and sequences of user actions related to the stages of the Purchase Decision Model (PDM) perform on par with the state-of-the-art LSTM model for sequential data. At the same time the classifiers are less computationally expensive and offer insights about highly predictive motifs and associated user actions. Our 
approach to feature construction leads to robust LR classifiers that perform well on truncated user sessions, providing reliable intent predictions for active sessions and support for live interventions. Our simulation results offer a strong baseline for future work on defining intervention policies and objective functions to be optimized through session interventions.

\section{ACKNOWLEDGEMENTS}

We wish to express our gratitude to JD.com for providing access to invaluable data and Hao Dong for his assistance. We also acknowledge the financial support from the International Doctoral Innovation Centre, Ningbo Education Bureau, Ningbo Science and Technology Bureau, and the University of Nottingham. This work was also supported by the UK Engineering and Physical Sciences Research Council [grant number EP/L015463/1].

\section{REFERENCES}

[1] [n. d.]. Comm100 Live Chat Benchmark Report 2018. https://www.comm100 com/livechat/resources/landing-live-chat-benchmark-report-2018/.

[2] [n. d.]. RecSys 2015. http://2015.recsyschallenge.com/. Accessed: 2010-09-30

[3] 2016. GDPR EU Regulations L 119/1. Official Journal of the European Union, http //eur-lex.europa.eu/legal-content/EN/TXT/PDF/?uri=CELEX:32016R0679. Accessed: 2018-05-22.

[4] Vladimir Barash, Natasa Milic-Frayling, and Marc A Smith. 2013. A Method for Discovering Dynamic Network Motifs by Encoding Topic Propagation. In Web Intelligence (WI) and Intelligent Agent Technologies (IAT), 2013 IEEE/WIC/ACM International foint Conferences on, Vol. 1. IEEE, 451-458.

[5] Christian Borgelt. 2005. Keeping things simple: finding frequent item sets by recursive elimination. In Proceedings of the 1st International Workshop on Open Source data mining: frequent pattern mining implementations. ACM, 66-70.

[6] Christian Borgelt. 2010. Simple Algorithms for Frequent Item Set Mining. Advances in Machine Learning II 263 (2010), 351-369.

[7] Mira Burri and Rahel Schär. 2016. The Reform of the EU Data Protection Framework. Journal of Information 6 (2016).

[8] Patrick Butler and Joe Peppard. 1998. Consumer purchasing on the Internet: Processes and prospects. European Management fournal 16, 5 (1998), 600-610.

[9] Yen-Liang Chen, Kwei Tang, Ren-Jie Shen, and Ya-Han Hu. 2005. Market basket analysis in a multiple store environment. Decision Support Systems 40, 2 (2005), 339-354.

[10] Zhicong Cheng, Bin Gao, and Tie-Yan Liu. 2010. Actively predicting diverse search intent from user browsing behaviors. In Proceedings of the 19th International Conference on World Wide Web. ACM, 221-230.

[11] Alex Graves and Jürgen Schmidhuber. 2005. Framewise phoneme classification with bidirectional LSTM and other neural network architectures. Neural Networks 18, 5-6 (2005), 602-610.

[12] Qi Guo and Eugene Agichtein. 2010. Ready to buy or just browsing?: detecting web searcher goals from interaction data. In Proceedings of the 33rd International ACM SIGIR Conference on Research and Development in Information Retrieval. ACM, 130-137.

[13] Sepp Hochreiter and Jürgen Schmidhuber. 1997. Long short-term memory. Neural Computation 9, 8 (1997), 1735-1780.

[14] David W Hosmer Jr, Stanley Lemeshow, and Rodney X Sturdivant. 2013. Applied logistic regression. Vol. 398. John Wiley \& Sons.

[15] John A Sheth Howard and N Jagdish. 1969. The theory of buyer behavior. Technical Report.

[16] Lele Kang, Xiang Wang, Chuan-Hoo Tan, and J Leon Zhao. 2015. Understanding the antecedents and consequences of live chat use in electronic markets. fournal of Organizational Computing and Electronic Commerce 25, 2 (2015), 117-139.

[17] Eunju Kim, Wooju Kim, and Yillbyung Lee. 2003. Combination of multiple classifiers for the customer's purchase behavior prediction. Decision Support Systems 34, 2 (2003), 167-175.

[18] Jong Woo Kim, Song-Yi Han, and Dong Sung Kim. 2012. Association rules application to identify customer purchase intention in a real-time marketing communication tool. In Ubiquitous and Future Networks (ICUFN), 2012 Fourth International Conference on. IEEE, 88-90.

[19] Kevin Lane Kotler, Philip Keller. 2015. Marketing Management, volume 14. Prentice Hall Englewood Cliffs.

[20] Philip Kotler. 1994. Marketing management, analysis, planning, implementation, and control, Philip Kotler. London: Prentice-Hall International.

[21] Dmitry Lagun, Mikhail Ageev, Qi Guo, and Eugene Agichtein. 2014. Discovering common motifs in cursor movement data for improving web search. In Proceedings of the 7th ACM International Conference on Web Search and Data Mining. ACM, 183-192.

[22] Weiqiang Lin, Pedro Saleiro, Natasa Milic-Frayling, and Eugene Ch'ng. 2018. Social media brand engagement as a proxy for E-commerce activities: a case study of Sina Weibo and JD. In 2018 IEEE/WIC/ACM International Conference on Web Intelligence (WI). IEEE, 375-382.

[23] Caroline Lo, Dan Frankowski, and Jure Leskovec. 2016. Understanding behaviors that lead to purchasing: A case study of Pinterest.. In In Proceedings of the 22nd ACM SIGKDD International Conference on Knowledge Discovery and Data Mining. 531-540.

[24] Christos Makris, Yannis Panagis, Evangelos Theodoridis, and Athanasios Tsakalidis. 2007. A web-page usage prediction scheme using weighted suffix trees. In International Symposium on String Processing and Information Retrieval. Springer, 242-253.

[25] Wendy W Moe. 2003. Buying, searching, or browsing: Differentiating between online shoppers using in-store navigational clickstream. fournal of Consumer Psychology 13, 1-2 (2003), 29-39.

[26] Alan L Montgomery, Shibo Li, Kannan Srinivasan, and John C Liechty. 2004. Modeling online browsing and path analysis using clickstream data. Marketing Science 23, 4 (2004), 579-595.

[27] Ashwin Paranjape, Austin R Benson, and Jure Leskovec. 2017. Motifs in temporal networks. In Proceedings of the Tenth ACM International Conference on Web Search and Data Mining. ACM, 601-610.

[28] Chanyoung Park, Donghyun Kim, Jinoh Oh, and Hwanjo Yu. 2015. Predicting User Purchase in E-commerce by Comprehensive Feature Engineering and Decision Boundary Focused Under-Sampling. In Proceedings of the 2015 International ACM Recommender Systems Challenge. ACM, 8.

[29] Nancy Pekala. 2017. How B2B Marketers are Leveraging Live Chat to Increase Sales. AMA Access: B2B Marketing, A business-to-Business Blueprint for Driving Sales.

[30] Peter Romov and Evgeny Sokolov. 2015. RecSys Challenge 2015: Ensemble learning with categorical features. In Proceedings of the 2015 International ACM Recommender Systems Challenge. ACM, 1.

[31] Ivan Ross. 1979. An information processing theory of consumer choice. fournal of Marketing (pre-1986) 43, 000003 (1979), 124

[32] Catarina Sismeiro and Randolph E Bucklin. 2004. Modeling purchase behavior at an e-commerce web site: A task-completion approach. Fournal of Marketing Research 41, 3 (2004), 306-323.

[33] Grażyna Suchacka and Grzegorz Chodak. 2017. Using association rules to assess purchase probability in online stores. Information Systems and e-Business Management 15, 3 (2017), 751-780.

[34] Tijmen Tieleman and Geoffrey Hinton. 2012. Lecture 6.5-rmsprop: Divide the gradient by a running average of its recent magnitude. COURSERA: Neural networks for machine learning 4, 2 (2012), 26-31.

[35] Efraim Turban, Jon Outland, David King, Jae Kyu Lee, Ting-Peng Liang, and Deborrah C Turban. 2017. Electronic Commerce 2018: A Managerial and Social Networks Perspective. Springer.

[36] Dirk Van den Poel and Wouter Buckinx. 2005. Predicting online-purchasing behaviour. European fournal of Operational Research 166, 2 (2005), 557-575.

[37] Jianyong Wang, Jiawei Han, and Chun Li. 2007. Frequent closed sequence mining without candidate maintenance. IEEE Transactions on Knowledge and Data Engineering 19, 8 (2007).

[38] Yu-Ting Wen, Pei-Wen Yeh, Tzu-Hao Tsai, Wen-Chih Peng, and Hong-Han Shuai. 2018. Customer purchase behavior prediction from payment datasets. In Proceedings of the Eleventh ACM International Conference on Web Search and Data Mining. ACM, 628-636.

[39] Ryen W White, Paul N Bennett, and Susan T Dumais. 2010. Predicting shortterm interests using activity-based search context. In Proceedings of the 19th ACM International Conference on Information and Knowledge Management. ACM, 1009-1018.

[40] Lizhen Xu, Jason A Duan, and Andrew Whinston. 2014. Path to purchase: A mutually exciting point process model for online advertising and conversion. Management Science 60, 6 (2014), 1392-1412.

[41] Jiang Yang, Lada A Adamic, and Mark S Ackerman. 2008. Competing to Share Expertise: The Taskcn Knowledge Sharing Community.. In The International AAAI Conference on Web and Social Media.

[42] Jinyoung Yeo, Sungchul Kim, Eunyee Koh, Seung-won Hwang, and Nedim Lipka. 2017. Predicting Online Purchase Conversion for Retargeting. In Proceedings of the Tenth ACM International Conference on Web Search and Data Mining. ACM, 591-600.

[43] Xiaohui Yu, Yang Liu, Xiangji Huang, and Aijun An. 2012. Mining online reviews for predicting sales performance: A case study in the movie domain. IEEE Transactions on Knowledge and Data Engineering 24, 4 (2012), 720-734. 